\title{
Robotic Transmitral Approach for Hypertrophic Cardiomyopathy With Systolic Anterior Motion
}

\author{
Nai-Kuan Chou, MD; Ryoi Okano, MD; Takeo Tedoriya, MD; I-Hui Wu, MD; \\ Hsi-Yu Yu, MD; Yih-Sharng Chen, MD; Ming-Jiuh Wang, MD; Nai-Hsin Chi, MD
}

\begin{abstract}
Background: Surgical intervention is indicated in symptomatic hypertrophic cardiomyopathy (HCM) patients with a ventricular outflow pressure gradient more than $50 \mathrm{mmHg}$. The transmitral approach, along with the transapical and transaortic approaches, is routinely used for myectomy, but all are open procedures. We describe a robotic transmitral approach that can be used to resolve septal hypertrophied muscle and eliminate mitral regurgitation (MR) using 1 cardiac incision.
\end{abstract}

\begin{abstract}
Methods and Results: We retrospectively analyzed 20 adult patients with obstructive HCM who exhibited concomitant severe MR and systolic anterior motion (SAM). The 2 groups comprised 12 standard full-sternotomy transaortic and 8 robotic transmitral approaches. The pre-intraventricular pressure gradient was $69 \pm 14.2 \mathrm{mmHg}$ in the robotic transmitral group and $70.2 \pm 17.4 \mathrm{mmHg}$ in the transaortic group $(P=0.876)$. Both groups had a similar left ventricular ejection fraction $(65 \pm 8 \%$ vs. $72 \pm 9 \%, P=0.901)$ and maximal ventricular wall thickness $(22.3 \pm 4.5$ and $21.7 \pm 6.0, P=0.835)$. Postoperative MR was reduced to less than grade II in all patients. In the robotic group, the postoperative pressure gradient was $1.5 \pm 2.6 \mathrm{mmHg}$, which was lower than that of the transaortic group at $10.6 \pm 10.8 \mathrm{mmHg}(\mathrm{P}=0.019)$. The cross-clamp time was $95.3 \pm 7.7 \mathrm{~min}$ in the robotic group and $104.7 \pm 20.8 \mathrm{~min}$ in the transaortic group $(P=0.193)$. The operation time was $237.5 \pm 22.4$ and $309.6 \pm 28.5 \mathrm{~min}(P<0.01)$ in the robotic transmitral and transaortic groups, respectively.
\end{abstract}

Conclusions: Using a robotic transmitral approach to treat with patients with HCM, SAM, and MR is feasible and reliable. Through 1 atrial incision, it is possible to resolve hypertrophy of the septum and eliminate both severe MR and SAM.

Key Words: Hypertrophic cardiomyopathy; Mitral valve regurgitation; Robotic surgery

H ypertrophic cardiomyopathy (HCM) exhibits variable disease severity and location of the left ventricular (LV) hypertrophy. ${ }^{1-3}$ Surgical intervention is indicated in symptomatic patients with a LV outflow tract (LVOT) pressure gradient $>50 \mathrm{mmHg},{ }^{1}$ and concomitant mitral valve surgery is required in $11-20 \%$ of patients undergoing myectomy. ${ }^{4}$

Dynamic outflow obstruction is caused by systolic anterior motion (SAM) of the anterior mitral leaflet, which results in complications for the patient because the downward anterior leaflet occupies the LVOT and the mitral valve becomes incompetent, aggravating the clinical outcome.

There are 3 major approach routes described for myectomy: transaortic (TA), ${ }^{5-7}$ transmitral (TM) ${ }^{8,9}$ and transapical. ${ }^{10,11}$ TA myectomy has been performed as a standard procedure since Morrow et al first described it in the $1960 \mathrm{~s} .{ }^{6}$ It became the most popular approach to excising hypertrophied LVOT muscle. The TM approach was described by Lillehei and Levy in $1963^{8}$ and is also a routinely used approach for some cardiac surgeons. The transapical approach is mainly used to excise hypertrophied muscle in the apical and mid-ventricular regions. ${ }^{10,11}$

In patients with a LVOT pressure gradient $>50 \mathrm{mmHg}$ plus SAM and severe mitral regurgitation (MR), our surgical aim is to first excise the hypertrophied septum in order to reduce the LVOT pressure gradient and then to resolve the SAM and eliminate MR.

We describe a robotic TM (RTM) approach to resolving septal hypertrophy muscle and eliminating MR using 1 cardiac incision and we compared the results using this approach with those of the open TA approach.

\section{Methods}

Retrospective Analysis

From 2000 to 2017, a total of 42 adult HCM patients underwent surgical treatment at the National Taiwan University Hospital; 10 had less than moderate MR and

Received December 14, 2017; revised manuscript received July 6, 2018; accepted July 31, 2018; released online September 26 , 2018 Time for primary review: 68 days

Department of Surgery (N.-K.C., R.O., I.-H.W., H.-Y.Y., Y.-S.C., N.-H.C.), Department of Anesthesiology (M.-J.W.), National Taiwan University Hospital and National Taiwan University College of Medicine, Taipei, Taiwan; Department of Cardiovascular Surgery, Ageo Central General Hospital, Saitama (R.O., T.T.), Japan

Mailing address: Nai-Hsin Chi, MD, National Taiwan University Hospital and National Taiwan University College of Medicine, No.7, Chung Shan S. Rd., Zhongzheng Dist., Taipei City 10002, Taiwan. E-mail: chinaihsin@gmail.com

ISSN-1346-9843 All rights are reserved to the Japanese Circulation Society. For permissions, please e-mail: cj@j-circ.or.jp 
only underwent TA myectomy, and the remaining 32 patients had concomitant severe MR and SAM. Of these 32 patients, 12 underwent mitral valve replacement by patient's choice and 20 underwent repair. Additionally, 8 underwent the RTM approach and the remaining underwent sternotomy with a TA approach and further transatrial mitral repair (Figure 1).

We compared the patients who underwent the RTM

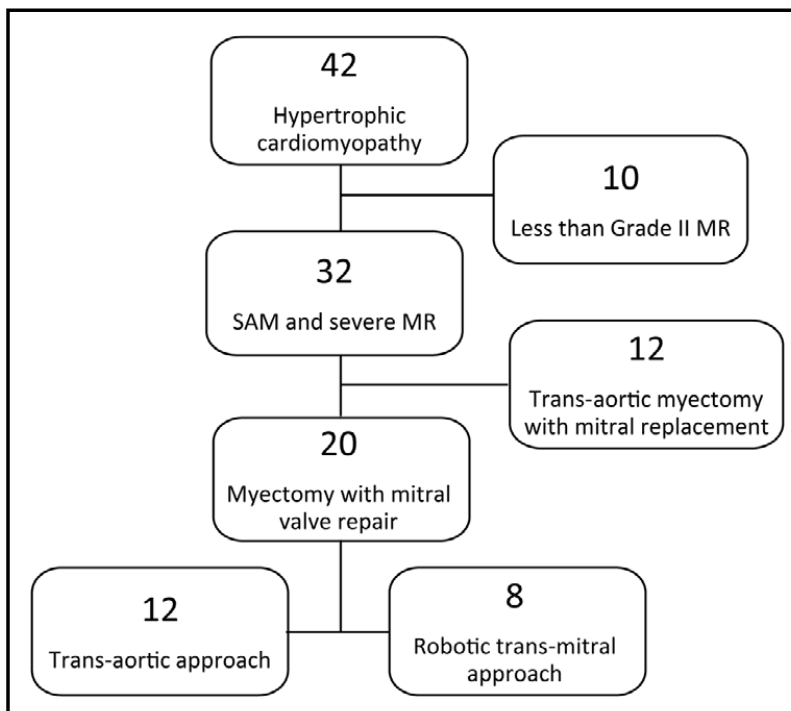

Figure 1. Patient enrollment: 42 adult HCM patients underwent surgical treatment; 10 were excluded for the less than moderate MR and the remaining 32 patients had concomitant severe MR and SAM. HCM, hypertrophic cardiomyopathy; MR, mitral regurgitation; SAM, systolic anterior motion. or TA approach. The pre- and postoperative pressure gradients and MR degree were compared.

\section{Indications for RTM}

The surgical indication for HCM and MR is patients with LVOT pressure gradient $>50 \mathrm{mmHg}$ and symptomatic despite medical treatment. The location and extension of the hypertrophic muscle is not a major concern for the robotic approach. Anywhere from the subaortic region, through the mid-ventricular area to the ventricular apex can be treated using RTM. The choice is essentially an economic one; if the patient can afford the robotic instruments fee, then we choose RTM; otherwise the surgery is conventional sternotomy and TA approach.

\section{Robotic Surgical Techniques and Settings (Figure 2)}

The working port and trocar ports are made first before heparinization. The $3-\mathrm{cm}$ working port is placed in the 4 th intercostal space around the right anterior axillary line. The right arm is inserted into the 6th intercostal space and the left arm into the 3 rd intercostal space. The left atrial retractor is inserted into the 4th intercostal space. Next, cardiopulmonary bypass is prepared with routine peripheral cannulation through the right femoral artery and vein. The right internal jugular vein is also cannulated to improve drainage. A cardioplegic needle and aortic cross-clamp are inserted through the working port, and antegrade cardioplegic solution is delivered. Routinely, a left atrial approach is adopted, with an incision through Sondergaard's plane. The mitral valve is exposed using an atrial retractor. We size the anterior leaflet first, using a sizing template (the template is the same size format as the Carpentier-Edwards Physio Annuloplasty Ring Sizer) (Figure 2A). Once the size is determined, we make a bovine pericardial patch using the template. The size also determines the posterior annuloplasty ring. We then take down the anterior mitral
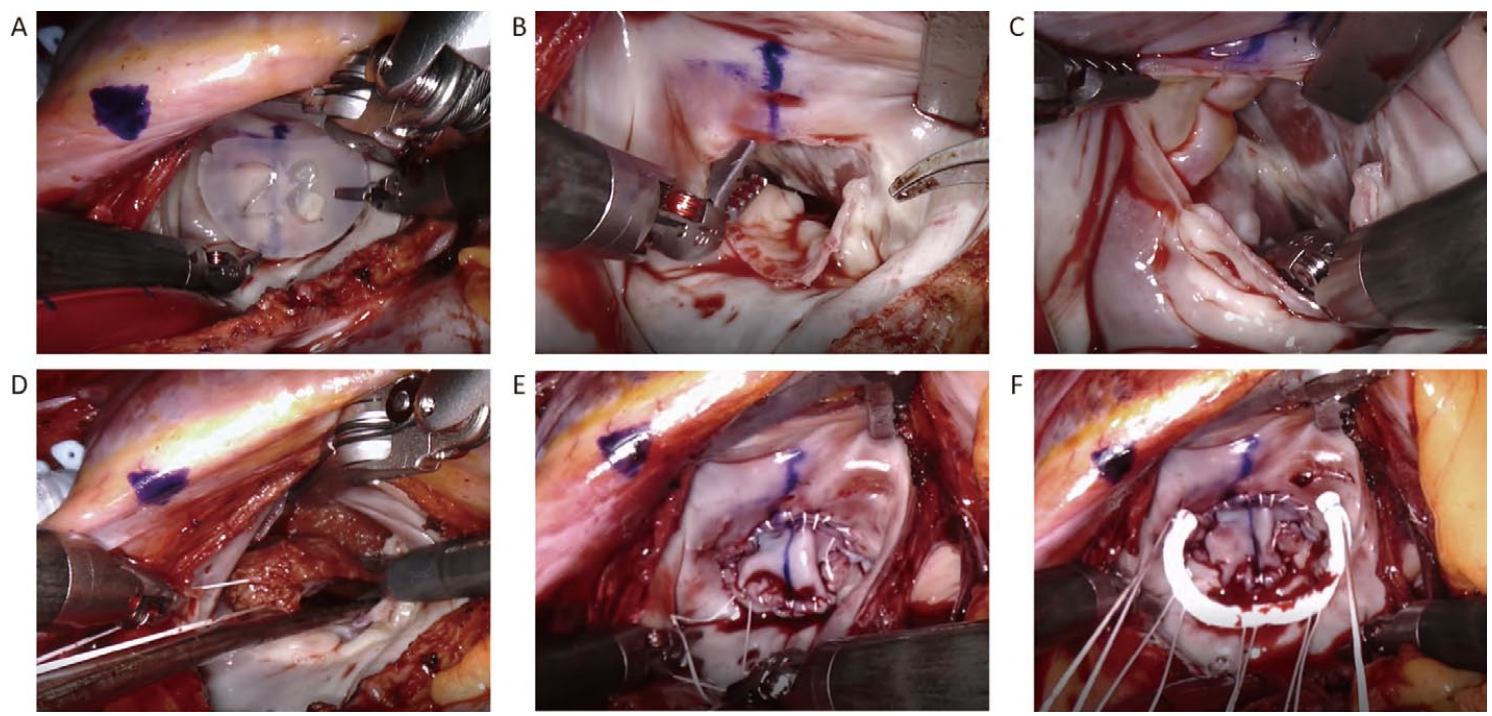

Figure 2. Steps in robotic transmitral surgery. (A) Anterior leaflet patch created as approximately the same size as the anterior leaflet template. (B) Anterior leaflet removal via resection from the anterolateral to the posteromedial commissure. (C) Checking the aortic valve and for jet lesions. (D) Removal of hypertrophied septal muscle. (E) Anterior leaflet augmentation by previously generated pericardial patch. (F) Posterior annulus fixation using an annuloplasty band. 


\begin{tabular}{|c|c|c|c|}
\hline Characteristics and variables & $\begin{array}{l}\text { RTM } \\
(n=8)\end{array}$ & $\begin{array}{c}\text { TA } \\
(n=12)\end{array}$ & $P$ value \\
\hline Age, years & $61.9 \pm 8.7$ & $57.2 \pm 11.6$ & 0.348 \\
\hline \multicolumn{4}{|l|}{ Sex } \\
\hline $\mathrm{M}, \mathrm{n}(\%)$ & $4(50)$ & $7(58.33)$ & 0.734 \\
\hline $\mathrm{F}, \mathrm{n}(\%)$ & $4(50)$ & $5(41.67)$ & \\
\hline Hypertension, n (\%) & $6(75)$ & $3(25)$ & 1.000 \\
\hline Diabetes mellitus, n (\%) & $6(75)$ & $5(41)$ & 0.462 \\
\hline NYHA functional class III or IV, n (\%) & $2(25)$ & $2(16.67)$ & 0.725 \\
\hline Syncope, n (\%) & $4(50)$ & $8(66.67)$ & 0.822 \\
\hline LVEF, n (\%) & $65 \pm 8$ & $72 \pm 9$ & 0.901 \\
\hline Maximum ventricular wall thickness, $\mathrm{mm}$ & $22.3 \pm 4.5$ & $21.7 \pm 6.0$ & 0.835 \\
\hline \multicolumn{4}{|l|}{$\mathrm{PG}, \mathrm{mmHg}$} \\
\hline Preoperation & $69 \pm 14.2$ & $70.2 \pm 17.4$ & 0.876 \\
\hline Postoperation & $1.5 \pm 2.6$ & $10.6 \pm 10.8$ & 0.019 \\
\hline \multicolumn{4}{|l|}{ MR grade } \\
\hline Preoperation & $3.5 \pm 0.5$ & $3.6 \pm 0.5$ & 0.734 \\
\hline Postoperation & $0.6 \pm 0.5$ & $0.9 \pm 0.5$ & 0.235 \\
\hline ICU stay, days & $3 \pm 2.5$ & $3 \pm 2.7$ & 0.870 \\
\hline Hospital stay, days & $15 \pm 48.7$ & $13 \pm 28.7$ & 0.737 \\
\hline
\end{tabular}

ICU, intensive care unit; LVEF, left ventricular ejection fraction; MR, mitral regurgitation; NYHA, New York Heart Association; PG, pressure gradient; RTM, robotic transmitral approach; TA, transaortic approach.

\begin{tabular}{|c|c|c|c|}
\hline Characteristics and variables & $\begin{array}{c}\text { RTM } \\
(n=8)\end{array}$ & $\underset{\substack{\text { TA } \\
(2)}}{ }$ & $P$ value \\
\hline \multicolumn{4}{|l|}{ Extent of hypertrophied muscle } \\
\hline Diffuse & 2 & 3 & \\
\hline Subaortic to mid-ventricle & 6 & 9 & \\
\hline \multicolumn{4}{|l|}{ Mitral valve pathology } \\
\hline No specific valve lesion & 5 & 8 & \\
\hline Abnormal chordae insertion & 2 & 3 & \\
\hline Chordae rupture & 1 & 1 & \\
\hline \multicolumn{4}{|l|}{ MVR procedure } \\
\hline Anterior augmentation+band annuloplasty & 8 & 3 & \\
\hline Edge-to-edge stitch+ring annuloplasty & 0 & 9 & \\
\hline Operation time, $\min$ & $237.5 \pm 22.4$ & $309.6 \pm 28.5$ & $<0.01$ \\
\hline Cross-clamp time, $\min$ & $95.3 \pm 7.7$ & $104.7 \pm 20.8$ & 0.193 \\
\hline
\end{tabular}

MVR, mitral valve repair; RTM, robotic transmitral approach; TA, transaortic approach.

leaflet (Figure 2B). The leaflet is detached from commissure to commissure, which allows the surgeon to evaluate the septum from the aortic annulus to the LV apex. The aortic valve is carefully checked to avoid injuring either it or the annulus; the presence of a jet lesion should also be checked (Figure 2C) and any lesions removed completely. The hypertrophied LV muscle, including the apical muscle, is removed completely using this approach (Figure 2D). Anterior leaflet is reattached to the annulus using bovine pericardial patch augmentation, which had been made previously (Figure 2E). The last step is to fix the posterior annulus using an annuloplasty band (Figure 2F).

\section{Statistical Analysis}

Numerical data are expressed as the mean \pm standard deviation. The frequencies of simple events are reported as simple percentages. Differences between groups were compared using Student's t-test, and the Shapiro-Wilke test.

\section{Results}

Table 1 lists the characteristics of both surgical groups. The average age of the patients was $61.9 \pm 8.7$ years in the RTM group and $57.2 \pm 11.6$ years in the TA group $(\mathrm{P}=0.348)$. In both groups, there were no differences in age, sex, or incidence of hypertension and diabetes. The preoperative intraventricular pressure gradient was $69 \pm 14.2 \mathrm{mmHg}$ and $70.2 \pm 17.4 \mathrm{mmHg}$ in the RTM and TA groups, respectively $(\mathrm{P}=0.876)$. Both groups of patients had a similar $\mathrm{LV}$ ejection fraction $(65 \pm 8 \%$ in RTM and $72 \pm 9 \%$ in TA, $\mathrm{P}=0.901)$ and a similar maximal ventricular wall thickness $(22.3 \pm 4.5 \mathrm{~mm}$ in $\mathrm{RTM}$ and $21.7 \pm 6.0 \mathrm{~mm}$ in TA, $\mathrm{P}=0.835$ ). There were no 

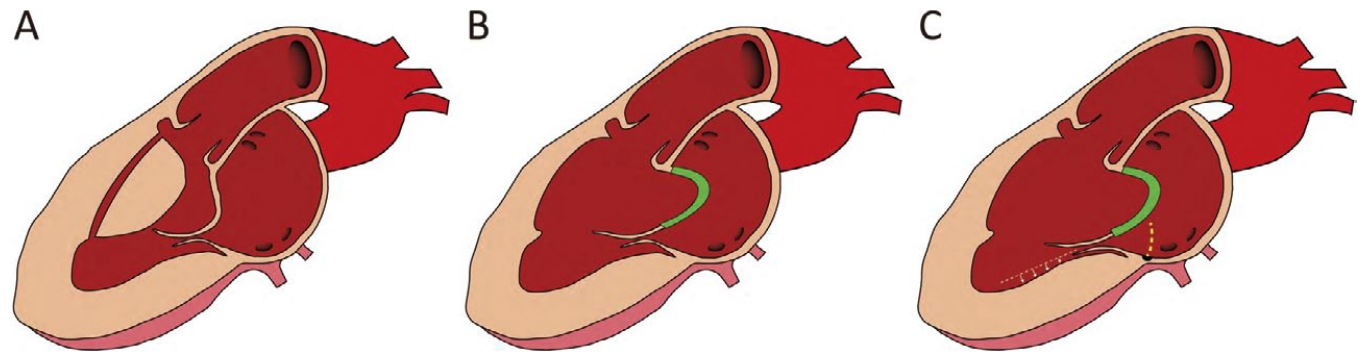

Figure 3. Mechanism of lowering the pressure gradient. (A) Hypertrophied septum is removed to increase the area of the ventricular outflow tract. (B) Green line indicates the bovine augmented pericardial patch. The anterior leaflet patch expands towards the left atrium during systole. (C) Yellow dotted line indicates the posterior partial band. The posterior band restrains posterior leaflet motion, making the coaption line (white dotted line) further posteriorly located (white arrows). This makes the ventricular outflow tract wider and further decreases the outflow pressure gradient.
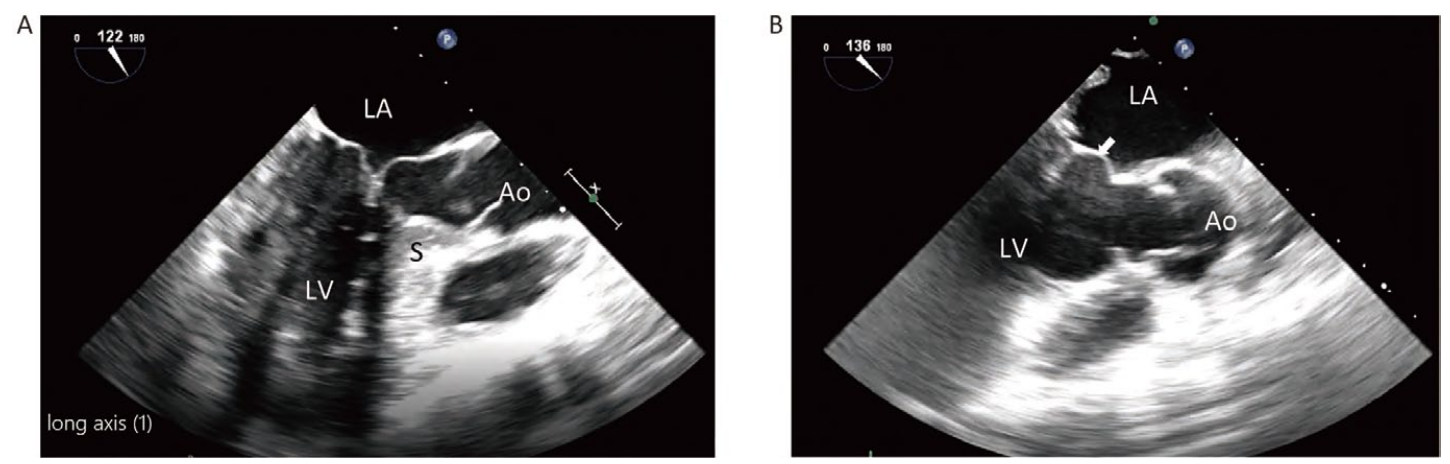

Figure 4. Transesophageal echocardiograms. (A) Long-axis view of the preoperative transesophageal echocardiogram. The hypertrophied septum and systolic anterior motion of the anterior leaflet obstruct the ventricular outflow tract. S, hypertrophied septum. (B) Long-axis view of the postoperative transesophageal echocardiogram. The outflow tract has opened, the septum has been removed, and the anterior leaflet patch (arrow) extends towards the left atrial side. Ao, aorta; LA, left atrium; LV, left ventricle.

operative deaths in either group. The preoperative MR was at least grade III in both groups, but reduced to less than grade II following surgery.

Hypertrophied muscle included the diffuse type and mid-ventricular type in both groups (Table 2). There were 3 cases of diffuse type in the TA group, and 2 in the RTM group; 9 cases of the mid-ventricular type in the TA group and 6 in the RTM group. The detailed mitral valve pathology is categorized in Table 2. In more than half of the patients, there was no specific valvular or subvalvular lesion. There were 2 patients with associated chordae rupture: one in each surgical group. This was repaired using neo-chord, and then anterior leaflet patch augmentation. There were 5 patients with abnormal chordae insertion: 3 in the TA and 2 in the RTM group. All the abnormal chordae were excised, followed by subsequent mitral repair procedures. In the TA group, 8 had the mitral valve repaired using edge-to-edge suture to plicate and fix the anterior leaflet anterior motion, and then the annulus was fixed by annuloplasty ring. The ring size was determined as for the anterior leaflet size according to most of the mitral valve repair rules. In the RTM group, the mitral valve repair method was anterior leaflet augmentation, and the annu- loplasty band fixation was over the posterior annulus. The size of posterior band was equal to the anterior leaflet size (true size) measured before taking down the anterior leaflet.

In the RTM group, the postoperative pressure gradient was $1.5 \pm 2.6 \mathrm{mmHg}$, which was lower than that of the TA group $(10.6 \pm 10.8 \mathrm{mmHg})(\mathrm{P}=0.019)$ (Table 1). The crossclamp time was $95.3 \pm 7.7$ and $104.7 \pm 20.8 \mathrm{~min}$ in the RTM and TA groups, respectively $(\mathrm{P}=0.193)$. The operation time was $237.5 \pm 22.4$ and $309.6 \pm 28.5 \mathrm{~min}$ in the RTM and TA groups, respectively $(\mathrm{P}<0.01)$ (Table 2$)$.

\section{Discussion}

As shown in the recruitment flowchart (Figure 1), the 12 patients who underwent TA myectomy and mitral valve replacement were excluded from our study group for comparative reasons. The reason those valves were not repaired was mostly surgeons' and patients' choices. Of the 10 surgeons at our institute, some were initially unfamiliar with repairing those valves in $\mathrm{HCM}$ patients.

The TA approach is sufficient for isolated HCM without SAM or MR, but limits the surgeon's ability to visualize the size of the aortic annulus. Surgeons acquire the requisite 
skill to excise hypertrophied muscle through the annulus down to ventricular apex, but if the patient has MR, which cannot be resolved from the TA approach, then the surgeon must make another left atrial incision to resolve both MR and SAM. Some patients can exhibit an abnormal submitral apparatus, such as abnormal papillary muscle insertion and abnormal chordae insertion, both of which should be removed to ensure surgical success.

Although the transapical approach has been described, the required incision may result in excessive bleeding and future apical akinesia, which can interfere with ventricular function. This approach is suitable in cases of mid-ventricular or apical hypertrophied muscle.

The TM approach was first described in $1963^{\circ}$ and facilitated surgical treatment because the mitral annulus is much larger than the aortic annulus, thereby enabling visualization of the submitral septum, even in the LV apex. However, the angle at which the surgery is performed sometimes requires surgeons to expose the mitral valve by applying traction on the atrial wall.

The RTM approach addresses these problems. The scope can pass through the mitral annulus, thereby allowing inspection of every portion of the septum. The septal area from the aortic annulus to the apex can be clearly exposed and jet lesions can be easily identified. Furthermore, by removing the anterior leaflet from commissure to commissure, abnormal submitral chordae or papillary muscle insertions can be excised. ${ }^{\mathbf{1 2}-14}$ In addition, this method increases the ability of the surgeon to visualize the submitral area. The hypertrophied muscle can be excised widely using this approach in order to decrease the LVOT pressure gradient. After removing all hypertrophied muscle, the anterior leaflet is repaired using a bovine pericardial patch. The patch size is measured to be approximately the same as that of the anterior leaflet using an annuloplasty ring sizer (true size of anterior leaflet). This augmentation of the anterior leaflet further guarantees the coaption zone and prevents SAM. Based on echocardiographic findings, the anterior leaflet patch actually goes to the left atrial side in the systolic phase (Figure 3B), which further increases both the area of the LVOT and the aorto-mitral angle (Figure 3C). Finally, the posterior mitral annulus is fixed using an annuloplasty band, the size of which is based on the measurement of the anterior leaflet's anterior-posterior diameter and intercommissural distance. A posterior annuloplasty band reduces the anterior-posterior mitral annular diameter and restrains the posterior leaflet, thereby allowing the line of coaptation to remain posteriorly located (Figure 3C) and further increases the LVOT area.

We also compared the pre- and postoperative cardiac echocardiographic findings (Figure 4). The preoperative LVOT was obstructed by the hypertrophied septum and worsened by SAM. The aorto-mitral angle was sharp and the LVOT was nearly completely obstructed (Figure 4A). The hypertrophied septum was removed during the operation and the anterior patch was extended to the left atrium to further increase the area of the LVOT and to eliminate the SAM (Figure 4B). As a result, the aorto-mitral angle flattened. We hereby propose a mechanism by which this RTM approach eliminates the LVOT pressure gradient, SAM, and MR.

1. Extended myectomy (Figure 3A): the hypertrophied muscle and jet lesion can be clearly identified. A wider myectomy can be performed through the submitral area, which gives the surgeon a better view towards the
LV apex. The LVOT can be opened using this method. 2. Anterior leaflet patching (Figure 3B): the patch not only augments the anterior leaflet, but also increases the aorto-mitral angle. During the systolic phase, the patch distends towards the left atrium, which further widens the LVOT. Using this patch, the mitral coaption area increases in size and eliminates MR because the LVOT pressure gradient disappears and the coaption area increases.

3. Posterior annulus band fixation (Figure 3C): after band fixation, the posterior leaflet shows a constrained motion, which moves the coaption area further away from the LVOT.

All 3 effects fully resolve the HCM pathology.

Both the RTM and TA approaches can effectively eliminate the pathological pressure gradient and establish a competent mitral valve. The pressure gradient in the RTM group was lower because the mitral patch method increased the space for the LVOT.

The cross-clamp times in both groups were not significantly different. The operation time was much less in the RTM group. The benefit of robotic surgery is that it requires a smaller incision without sternotomy. However, there are small trocar holes in the right thoracic cage following this procedure. If performed by an experienced robotic cardiac surgeon, the operation time could be well controlled. This study had some limitations, including that all patients in the RTM group were treated by a single surgeon.

\section{Conclusions}

Using the RTM approach to treat with patients with HCM, SAM, and MR was safe and reliable. It was possible to resolve hypertrophy of the septum and eliminate both the severe MR and the SAM through 1 incision.

\section{Acknowledgments}

The authors thank the staff at the Seventh Core Laboratory, Department of Medical Research of the National Taiwan University Hospital for their technical support. The authors also thank Liou Yi-Jia for help with statistical analysis and figure development. The program was partially funded by the Ministry of Science and Technology of Taiwan (MOST 105-2314-B-002-128).

\section{Conflict of Interest}

None.

\section{References}

1. JCS Joint Working Group. Guidelines for diagnosis and treatment of patients with hypertrophic cardiomyopathy (JCS 2012): Digest version. Circ J 2016; 80: 753-774.

2. Kitaoka H, Kubo T, Okawa M, Hitomi N, Furuno T, Doi YL. Left ventricular remodeling of hypertrophic cardiomyopathy longitudinal observation in a rural community. Circ J 2006; 70: $1543-1549$

3. Matsumori A, Furukawa Y, Hasegawa K, Sato Y, Nakagawa H, Morikawa Y, et al. Epidemiologic and clinical characteristics of cardiomyopathies in Japan: Results from nationwide surveys. Circ J 2002; 66: 323-336.

4. Yu EH, Omran AS, Wigle ED, Williams WG, Siu SC, Rakowski $\mathrm{H}$. Mitral regurgitation in hypertrophic obstructive cardiomyopathy: Relationship to obstruction and relief with myectomy. $J$ Am Coll Cardiol 2000; 36: 2219-2225.

5. Binaco I, Spirito P, Poggio D, Casati V, Grillo M, Ferrazzi P. Transaortic mitral valve secondary chordal cutting in patients with obstructive hypertrophic cardiomyopathy and mild septal hypertrophy. Ann Cardiothorac Surg 2017; 6: 426-428.

6. Morrow AG. Hypertrophic subaortic stenosis: Operative methods utilized to relieve left ventricular outflow obstruction. $J$ 
Thorac Cardiovasc Surg 1978; 76: 423-430.

7. Ralph-Edwards A, Vanderlaan RD, Bajona P. Transaortic septal myectomy: Techniques and pitfalls. Ann Cardiothorac Surg 2017; 6: $410-415$.

8. Lillehei C, Levy MJ. Transatrial exposure for correction of subaortic stenosis. JAMA 1963; 186: 8-13.

9. Meier S, Noack T, Mohr FW, Seeburger J, Passage J. Transmitral myectomy and how to deal with systolic anterior motion (SAM) in hypertrophic obstructive cardiomyopathy. Ann Cardiothorac Surg 2017; 6: 416-418.

10. Kotkar KD, Said SM, Schaff HV. Transapical approach for myectomy in hypertrophic cardiomyopathy. Ann Cardiothorac Surg 2017; 6: 419-422.

11. Said SM, Schaff HV, Abel MD, Dearani JA. Transapical approach for apical myectomy and relief of midventricular obstruction in hypertrophic cardiomyopathy. J Card Surg 2012; 27: $443-448$.

12. Chung YS, Cho I, Park B, Hong J. An abnormal chord inserting to a subaortic membrane in hypertrophic cardiomyopathy: Rare anatomical combination. Eur J Cardiothorac Surg 2017; 52: 1008.
13. Wang S, Cui H, Yu Q, Chen H, Zhu C, Wang J, et al. Excision of anomalous muscle bundles as an important addition to extended septal myectomy for treatment of left ventricular outflow tract obstruction. J Thorac Cardiovasc Surg 2016; 152: $461-468$.

14. Nomura T, Harada Y, Suzaki Y, Hayashi H, Tanaka H, Shiraishi J, et al. Left ventricular outflow tract obstruction due to anomalous insertion of papillary muscle. Circ J 2004; 68: $1219-1222$

\section{Supplementary Files}

\section{Supplementary File 1}

Movie S1. A 60-year-old man diagnosed with hypertrophic cardiomyopathy and severe mitral regurgitation. The pressure gradient over the ventricular outflow tract was $90 \mathrm{mmHg}$. We performed a transmitral myectomy and patch augmentation of the anterior mitral leaflet using a robotic approach.

Please find supplementary file(s);

http://dx.doi.org/10.1253/circj.CJ-17-1369 